\title{
INVESTIGACIONES
}

\section{Teorías implícitas del profesorado de formación profesional para el empleo}

\author{
Implicit theories of Vocational Education and Training teachers
}

\author{
Alicia Ros-Garrido, ${ }^{a}$ Almudena Navas-Saurin, ${ }^{a b}$ Fernando Marhuenda Fluixáa \\ a'Departament de Didàctica i Organització Escolar, Universitat de València \\ Telf.: (34) 963864427. Correo electrónico: Alicia.Ros@uv.es \\ bTelf.: (34) 963864427. Correo electrónico: Almudena.Navas@uv.es \\ cTelf.: (34) 963864595. Correo electrónico: Fernando.Marhuenda@uv.es
}

\begin{abstract}
RESUMEN
Este artículo aborda las teorías implícitas del profesorado de Formación Profesional para el Empleo con el objetivo de comprender en qué medida son dispares las teorías implícitas del profesorado de la modalidad Formación e inserción y la modalidad Colectivos. La investigación realizada es de orientación cuantitativa basada en un método descriptivo. El instrumento utilizado es la adaptación del "Cuestionario de teorías implícitas del profesorado sobre la enseñanza" (Marrero, 1988a). El análisis de contenido de la estructura resultante de los análisis factoriales realizados permite establecer tres orientaciones de la enseñanza con sentido teórico que permiten concluir que las teorías implícitas u orientaciones de la enseñanza del profesorado de Formación Profesional para el Empleo son diferentes dependiendo del colectivo al que van dirigidas las acciones formativas.
\end{abstract}

Palabras clave: teorías implícitas, formación profesional, formación para el empleo, programas de empleo.

\begin{abstract}
This article discusses implicit theories upon teaching of Vocational Education and Training teachers. The aim is to understand to what extent implicit theories of teachers vary between those working in the 'training' modality and those working in the 'addressee' modality. Our research is quantitative and descriptive. We have adapted, for the purpose of our research, the "Questionnaire of implicit theories of teachers on teaching" (Marrero, 1988a). The content analysis of the resulting structure of the factorial analysis made it possible to identify, in our sample, three orientations to teaching in Vocational Training Programs. We have been able to conclude that implicit theories or orientation towards teaching of these trainers are different depending on the beneficiaries to which the training offer is addressed.
\end{abstract}

Key words: implicit theories, vocational education, job training, employment programs. 


\section{INTRODUCCIÓN}

La Formación Profesional ha sido poco atendida desde la academia (Merino, 2012; OCDE, 2011), a pesar de estar "llamada a ser una herramienta de cambio y mejora social" (Marhuenda, 2012, p. 12), y pasar a ser una alternativa renovada de formación (Álvarez et al., 2015). En este contexto, la Formación Profesional para el Empleo (FPE) ha recibido mucha menos atención y menos aún el profesorado que la imparte (Ruiz, 2001). Ante esta realidad, el objeto de estudio constituye una aproximación a un contexto escasamente abordado en el ámbito académico y sobre el que es necesario profundizar.

El contexto concreto de investigación forma parte de la denominada formación de oferta de acuerdo a lo establecido en el Real Decreto 395/2007 por el que se regula el subsistema de FPE español y que, entre otros aspectos, integra por fin las llamadas formación ocupacional y formación continua en un único subsistema. Las modalidades objeto de estudio forman parte de los programas formativos de FPE cofinanciados por el Fondo Social Europeo y dirigidos, prioritariamente, a personas en situación de desempleo, a fin de proporcionarles una cualificación profesional para desempeñar una ocupación adecuada a las necesidades del mercado de trabajo y estimular su contratación o inserción laboral.

Las incertidumbres del mercado de trabajo actual provocan que no siempre la FPE responda a necesidades reales de las demandas sociolaborales y a la necesidad de desarrollar competencias básicas en sus participantes (Olmos, 2014), lo que a su vez produce cierto desajuste entre la oferta y la demanda de empleo. De ahí que sea necesario un ajuste mediante un estudio de necesidades reales (Buendía \& Berrocal, 2008; Buendía, Expósito \& Sánchez, 2012). Esta realidad necesariamente nos aboca a tener presente el profesorado que la imparte, puesto que debe estar en contacto con el contexto de producción o realidad profesional del contexto laboral tal y como ha demostrado el estudio realizado por Barrientos y Navío (2015).

En el contexto valenciano, desde la Orden 29/2012, de 22 de junio de la Conselleria de Educación, Formación y Empleo, por la que se determina el Programa de FPE y se regulan y convocan subvenciones para la realización de acciones formativas dirigidas prioritariamente a las personas trabajadoras desempleadas durante el ejercicio 2012, se han establecido cuatro modalidades de programación: Formación e inserción (o para la inserción), Semipresencial, Formación a medida y Colectivos.

En la investigación realizada ha participado profesorado de la modalidad Formación e inserción y de la modalidad Colectivos. La primera se dirige prioritariamente a personas desempleadas en general, aunque también pueden participar personas trabajadoras ocupadas. La segunda se dirige a personas desempleadas con alguna discapacidad (Colectivos-DIS) o en riesgo de exclusión social (Colectivos-RES) y viene a sustituir a los anteriormente denominados Talleres de Formación e Inserción Laboral (Talleres FIL) con la principal diferencia de que desde la convocatoria de 2012 hacen referencia a certificados de profesionalidad ${ }^{1}$.

\subsection{LAS TEORÍAS IMPLÍCITAS}

Para Angulo (1999) y para Marrero $(2009,2010)$ las investigaciones desarrolladas sobre el conocimiento docente pueden agruparse en cuatro grupos: centradas en la planificación,

Es un documento que acredita a un trabajador/a en una cualificación profesional del Catálogo Nacional de las Cualificaciones Profesionales en España. 
centradas en la toma de decisiones, centradas en las teorías o creencias y centradas en la diferencia entre profesorado novato y experto. El trabajo aquí expuesto forma parte de las investigaciones centradas en las teorías o creencias del profesorado, y tienen especial relevancia para este estudio las investigaciones realizadas por Marrero (1988a, 1988b, 1991, 1992) y Rodrigo, Rodríguez y Marrero (1993) donde se conceptualiza las teorías de la enseñanza como conocimiento representacional y las teorías implícitas como conocimiento atribucional. La diferencia entre los dos tipos de conocimiento radica en la tarea que el sujeto tiene que afrontar (no en el contenido), es decir, es una tarea teórica para las síntesis representacionales o una tarea pragmática para las atribucionales, "Según el tipo de demanda, varía la estructura interna de la síntesis, así como su valor instrumental" (Jiménez, 2005, p. 214). Dado que en todo conocimiento hay componentes implícitos y explícitos (Makuc, 2015), es interesante la diferencia entre teorías implícitas y explícitas sobre el aprendizaje y la enseñanza que realizan Pozo et al. (2006), donde afirman que dichas teorías se adquieren por procesos de aprendizaje implícito o explícito (respectivamente). El contexto o escenarios culturales de las personas determinan que del conjunto de teorías disponibles a nivel representacional "sólo algunas pasen a tener entidad atribucional" (Jiménez \& Correa, 2002, p. 531).

Tras la revisión teórica realizada, consideramos que las teorías implícitas o conocimiento atribucional del profesorado no son una reproducción lineal ni una recuperación mecánica ni una traslación pasiva de las teorías de la enseñanza (síntesis de conocimientos), sino una síntesis coherente de creencias que el sujeto asume como propias y que conforman su concepción de la enseñanza y que está condicionada por las demandas de la tarea e influida por los contextos próximos de referencia (Correa \& Camacho, 1993; Correa \& Marrero, 1992; Jiménez, 2002, 2009; Makuc, 2008, 2011; Marrero, 1988a, 1991, 1992, 1993, 2009, 2010; Rodrigo et al., 1993). Las teorías implícitas son un conocimiento durable, fácilmente recuperable y difícil de cambiar (Gómez \& Guerra, 2012; Kagan, 1992) puesto que están influenciadas por el modo en que el profesorado se formó (Oramas, 2013) y se transmiten durante su proceso de formación (Makuc, 2011). Algunos autores consideran que la experiencia docente no cambia la teoría implícita asumida en la etapa escolar (Alarcón \& Reyno, 2009; Rodríguez, 2003), mientras que otros defienden que sí puede cambiar lentamente debido a procesos de socialización o al desarrollo profesional (Marrero, 1991), o después de un periodo de prácticas (Cepeda, 2009). En síntesis, las teorías implícitas combinan información procedente de diversas fuentes como el entorno sociocultural, la formación escolar o la experiencia (Makuc, 2015).

Si las teorías implícitas son "síntesis de experiencias pedagógicas" (Marrero, 2009, p. 37), las teorías implícitas del profesorado de FPE son síntesis de experiencias pedagógicas que se construyen, entre otros, en el escenario de la propia FPE y en contextos profesionales o entornos de trabajo.

\section{DISEÑO Y METODOLOGÍA}

La presente investigación es una aplicación de los estudios sobre el conocimiento docente, que gozan ya de una tradición consolidada, al contexto de la FPE. Su objetivo es comprender en qué medida son dispares las teorías implícitas del profesorado de la modalidad Formación e inserción y la modalidad Colectivos en el contexto de la provincia de Valencia. 


\subsection{DISEÑO E INSTRUMENTO}

La investigación realizada es de orientación cuantitativa basada en un método descriptivo. El instrumento utilizado es un cuestionario que incluye datos sobre la acción formativa, datos sobre la vinculación a la entidad en la acción formativa señalada, preguntas sobre el currículum en la práctica respecto al colectivo destinatario, datos personales y profesionales y sobre la trayectoria y experiencia laboral del profesorado ${ }^{2}$. El orden de los apartados anteriores ha seguido las recomendaciones de Azofra (1999) para cuestionarios amplios.

Para el estudio de las teorías implícitas se adaptó el "Cuestionario de teorías implícitas del profesorado sobre la enseñanza” (Marrero, 1988a) para evitar el empleo de un lenguaje sexista y adaptarlo al contexto de la FPE. En la adaptación también se tenía como referencia las investigaciones realizadas por Ferrández et al. (2000) y Molpeceres, Chulvi y Bernad (2004). El cuestionario fue validado por dos personas expertas en FPE, con casi 10 años de experiencia tanto como formadoras como gestoras en varias entidades formativas. Está constituido por 33 ítems que integran las cinco teorías implícitas identificadas por Marrero (1988a): la teoría dependiente (síntesis de la teoría tradicional) está compuesta por 7 ítems y se caracteriza por un profesorado obsesionado por el contenido, que guía y dirige la enseñanza con un ritmo constante y una actitud distante con el alumnado, al que considera que, por sí solo, no es capaz de aprender; la teoría productiva (síntesis de la teoría técnica) está compuesta por 6 ítems y considera la enseñanza como la búsqueda de resultados, la obsesión es por los objetivos en búsqueda de eficacia y concibe la evaluación como mecanismo selectivo y de control; la teoría expresiva (síntesis de la teoría activa) está compuesta por 6 ítems y responde al principio de actividad donde prima la ocupación permanente del alumnado en clase; la teoría interpretativa (síntesis de la teoría constructiva) está compuesta por 7 ítems y es una pedagogía centrada en el alumnado (en sus necesidades, recursos y procesos de aprendizaje) y donde prima la búsqueda de explicaciones de las prácticas y los procesos frente a los resultados; la teoría emancipatoria (síntesis de la teoría crítica) está compuesta por 7 ítems y tiene un marcado carácter moral y político, buscando alternativas moralmente coherentes y cuyas prácticas de enseñanza están vinculadas al marco político y social.

El formato de respuesta es mayoritariamente cerrado de elección de una opción, y en el caso del cuestionario de teorías implícitas el formato es una escala de respuesta para cada enunciado tipo Likert con 7 puntos de anclaje desde 1 (=estoy totalmente en desacuerdo) hasta 7 (=estoy totalmente de acuerdo).

\subsection{POBLACIÓN Y MUESTRA}

Respecto a la población, la investigación se ha dirigido a toda la población: profesorado que impartía las especialidades formativas que podían solicitarse de acuerdo a la convocatoria de la Orden 29/2012, de 22 de junio, por la cual se determina el Programa de FPE durante el ejercicio de 2012 (y que continuaban desarrollándose en 2013) y que coincidían tanto en la modalidad Formación e inserción como en la modalidad Colectivos en la provincia de Valencia.

Las cuestiones concretas objeto de investigación pueden consultarse en Ros-Garrido (2014). 
De acuerdo a lo establecido en la normativa, se estima que la modalidad Formación e inserción la imparte un profesor/a, mientras que en el caso de la modalidad Colectivos la imparten al menos dos profesores/as. Así, en las especialidades formativas seleccionadas, los datos de la población de referencia y la muestra obtenida ${ }^{3}$ son

- $\quad$ En la modalidad Formación e inserción se han concedido un total de 69 cursos. Población estimada: 69 docentes. Muestra obtenida: 41 docentes, lo que supone el $59,42 \%$ de la población.

- $\quad$ En el caso de la modalidad Colectivos-RES, se han concedido 7 cursos. Población estimada: 14 docentes. Muestra obtenida: 6 docentes, lo que supone el 42,86\% de la población.

- $\quad$ En el caso de la modalidad Colectivos-DIS, se han concedido 6 cursos. Población estimada: 12 docentes. Muestra obtenida: 8 docentes, lo que supone el 66,67\% de la población.

La población total de referencia es aproximadamente de 95 docentes. Consideramos que la muestra de 55 docentes es aceptable para el objeto de estudio, porque la muestra equivale aproximadamente al 58\% de la población.

\subsection{PROCEDIMIENTO DE RECOGIDA DE DATOS}

En el procedimiento de recogida de datos se pueden establecer los siguientes pasos. En primer lugar el envío de una carta personalizada a entidades formativas a las que se les había concedido acciones formativas subvencionadas objeto de estudio. En segundo lugar el contacto telefónico con la persona responsable de la gestión de la FPE en dicha entidad. Se hacía referencia a la carta enviada, se resolvían dudas sobre la investigación y se solicitaba su colaboración para remitir el cuestionario al profesorado que impartía dichas acciones formativas. En tercer lugar, el envío de los cuestionarios era inmediatamente posterior al contacto telefónico, se realizaba por correo electrónico y se codificaba para poder identificar la entidad a la que hacía referencia y, al mismo tiempo, se garantizaba la confidencialidad de los datos extraídos y el anonimato de los informantes. El formato del cuestionario permitía guardar las respuestas en el mismo documento y, una vez cumplimentado, poder enviarlo cumplimentado por correo electrónico a la investigadora. En cuarto lugar la recepción de cuestionarios cumplimentados y revisión del cumplimiento de los criterios establecidos, a la vez que se contactaba de nuevo con las entidades cuyos docentes no enviaban los cuestionarios.

El periodo fue el comprendido desde el 22 de febrero al 14 de mayo de 2013.

\footnotetext{
3 En total se recibieron 74 cuestionarios cumplimentados por docentes, pero algunos no se tuvieron en cuenta por no ser las especialidades formativas o las modalidades de formación objeto de investigación (de acuerdo a la información proporcionada por la Subdirección General de FPE) o no estar debidamente cumplimentados (preguntas sin contestar, con puntuaciones extremas o sin graduar la respuesta). Este último motivo responde a las recomendaciones de Marrero (1988a) y Correa y Camacho (1993): los sujetos extremos y los que dan la misma respuesta en casi todas los enunciados no deben tenerse en cuenta para proceder al análisis del cuestionario de teorías implícitas.
} 


\section{RESULTADOS}

En este apartado se exponen los resultados obtenidos en la presente investigación según su carácter estadístico y a continuación se muestra el resumen de las características significativas según los residuos tipificados corregidos (en adelante, RTC) en cada orientación de la enseñanza identificada, prestando especial atención a las diferencias según la modalidad en la que imparte el profesorado de FPE. El apartado finaliza con los resultados según su carácter teórico.

\subsection{RESULTADOS ESTADÍSTICOS}

Respecto a los resultados estadísticos, las dimensiones identificadas por Marrero (1988a) para el contexto escolar han resultado no ser válidas para el contexto de la FPE: la fiabilidad según el Alfa de Cronbach (en adelante, $\alpha$ ) para analizar la consistencia interna de los ítems que componen cada dimensión de las teorías implícitas no es aceptable (dependiente $\alpha=.525$; productiva $\alpha=.315$; expresiva $\alpha=.634$; interpretativa $\alpha=.455$; y emancipatoria $\alpha=.478$ ) puesto que para ser considerada aceptable los valores deberían estar entre .7 y .9 (Campo-Arias \& Oviedo, 2008).

La falta de normalidad de los ítems (con una significatividad entre ,000 y ,005 en la prueba de Kolmogorov-Smirnov) y que ningún sujeto aparezca polarizado (según los valores establecidos por Correa y Camacho (1993): entre -,06 y ,46) llevan a confirmar que el profesorado de FPE no se identifica con una única teoría, puesto que comparte ideas de varias teorías/factores y será necesario identificar nuevas agrupaciones o dimensiones en la escala de las teorías implícitas más acordes con el contexto de la FPE.

Ante estos resultados, consideramos pertinente realizar análisis factoriales con carácter exploratorio para realizar una síntesis de la información o reducción de la dimensión (número de variables) y poder obtener nuevos componentes principales o factores que sean una combinación lineal de las variables iniciales que expliquen la mayor parte de su variabilidad y sean un resumen de las variables con las que estén más claramente correlacionadas (Pérez, 2005). Se pretende obtener unos factores o componentes con mayor fiabilidad para la muestra analizada y que constituyan un marco teórico para la interpretación de las teorías implícitas del profesorado de FPE. Los resultados de los análisis factoriales muestran que los ítems están relacionados y que el análisis factorial no es apropiado para el estudio de las variables que forman el cuestionario de teorías implícitas del profesorado sobre la enseñanza. Entonces, al igual que Molpeceres et al. (2004), decidimos aplicar el análisis de componentes principales (ACP) con carácter exploratorio, puesto que teniendo en cuenta la naturaleza de las variables (las puntuaciones oscilan entre 0 y 7 ), es necesario el empleo de técnicas que no amortigüen las relaciones o agrupaciones de variables. Correa y Camacho (1993) afirman que

Hemos utilizado el ACP y no el análisis factorial (AF) porque, a igualdad de condiciones, el ACP obtiene pesos mayores. Esto se debe a que en el ACP se diagonaliza una matriz con unos en la diagonal y en el AF se diagonaliza una matriz con coeficientes de correlación o regresiones múltiples en la diagonal, que normalmente son menores que uno. Dada la naturaleza de las variables, necesitamos técnicas que no amortigüen el descubrimiento de relaciones o agrupaciones de variables. (Correa \& Camacho, 1993, pp. 148-149). 
$\mathrm{Al}$ argumento anterior, Jiménez (2005) añade las siguientes razones para elegir esta técnica:

a) Está orientada a explicar la mayor proporción de varianza de las variables originales mediante el menor número posible de componentes (Batista, 1984).

b) Supone que buscamos simplificar la estructura de datos sin hipotetizar ningún modelo «a priori» (Cuadras, 1991).

c) Una aplicación usual del ACP es tratar de establecer la dimensionalidad latente de un conjunto de variables originales, discriminando lo esencial y prescindiendo de la información redundante que existe en las n variables originales (Batista, 1984). (Jiménez, 2005, p. 226).

Se realizaron varios ACP con carácter exploratorio (forzando a 5, 4 y 3 tres factores), utilizando el método con rotación varimax a fin de verificar la estructura subyacente de los ítems de las teorías implícitas. Previamente se estudió el índice de adecuación de Kaiser-MeyerOlkin (KMO). En todos los casos, el test de esfericidad de Bartlett fue significativo al nivel de $p=.000$, y se utilizó una saturación de .30 como criterio para la inclusión del ítem en el factor.

Si bien puede pensarse que la estructura "ideal" sería de cinco componentes (Jiménez \& Correa, 2002), los ACP realizados abocan a tres componentes o factores. Observando el grado de saturación de cada ítem con cada componente o factor, la fiabilidad de los factores en el caso de incluir ítems que saturan en varios factores y el índice de comunalidad de dichos ítems, se toma la decisión informada de excluir nueve ítems de esta estructura factorial por no tener una clara relación con algún factor, por lo que no se han tenido en cuenta para posteriores análisis ${ }^{4}$. El índice de adecuación KMO de la estructura de 3 factores ha arrojado un valor de 0.318 y el test de esfericidad de Bartlett fue significativo al nivel de $\mathrm{p} \leq .000(\chi 2=937,894 ; \mathrm{gl}=528)^{5}$. Así, los tres factores con autovalores mayores de 2.589 dan cuenta de un $32.278 \%$ de la varianza en las puntuaciones: (a) primer factor da cuenta de un 14,307\% de la variación (9 ítems, autovalor=4.721, a=.769); (b) segundo factor explica un 10.124\% de la varianza (6 ítems, autovalor=3.341, a=.617); y (c) tercer factor supone un $7.846 \%$ de la varianza ( 7 ítems, autovalor=2.589, a=.621). La estructura resultante es la que aparece en la Tabla 1.

Tabla 1. Características de los factores identificados

\begin{tabular}{|c|c|c|c|}
\hline & Factor 1 & Factor 2 & Factor 3 \\
\hline $\mathrm{a}$ & .771 & .673 & .621 \\
\hline $\mathrm{N}^{\mathrm{o}}$ ítems & 9 & 8 & 7 \\
\hline $\begin{array}{c}\text { Ítems según Marrero } \\
(1988 \mathrm{a})\end{array}$ & $\begin{array}{c}32,27,26,13,1,29, \\
16,30 \mathrm{y} 25\end{array}$ & $\begin{array}{c}11,12,19,5,4,10,9 \\
\mathrm{y} 28\end{array}$ & $\begin{array}{c}3,6,21,2,14,20 \mathrm{y} \\
22\end{array}$ \\
\hline
\end{tabular}

Fuente: a partir de Ros-Garrido (2014).

El valor del índice de comunalidad oscila entre 0 y 1 y permite identificar los ítems que pueden eliminarse de la estructura factorial por su baja comunalidad $(<0,50)$, puesto que no son explicados por los componentes (Correa \& Camacho, 1993).

5 Los datos obtenidos del estadístico KMO no se acercan a la unidad, lo que indica que no es la adecuación deseable de nuestros datos a un modelo de análisis factorial (Pérez, 2005). No obstante, el contraste de Bartlett dice que no es significativa la hipótesis nula de variables iniciales incorrelacionadas (Pérez, 2005), por lo tanto decidimos aplicar el ACP con carácter exploratorio. 
Los resultados del ACP muestran, al igual que en los análisis factoriales, que los ítems están relacionados y que el ACP no es apropiado para el estudio de las variables que forman el cuestionario de teorías implícitas del profesorado sobre la enseñanza. No obstante, se decide profundizar en los resultados.

Constatamos, pues, que los valores de fiabilidad obtenidos son mejores que si se tomaran las dimensiones identificadas por Marrero (1988a). Antes de profundizar en el sentido teórico de los factores identificados, se observa la distribución de la muestra en los mismos. Atendiendo al promedio de la suma de la media de los ítems de cada nuevo factor, el profesorado participante en la investigación está más de acuerdo con los enunciados del primer factor, con un promedio de 5,98 sobre 7. Entre los otros dos factores hay poca diferencia: para el segundo factor el promedio es de 3,33 y para el tercer factor de 3,95. El análisis de conglomerados ${ }^{6}$ reafirma los resultados, puesto que tanto los sujetos como las variables se agrupan en un único clúster, es decir, se asume en mayor medida el primer factor.

Se toma la decisión de comprobar el grado de asunción de los nuevos factores, es decir, comprobar si el profesorado de la muestra asume exclusivamente un factor o si comparte ideas de varios factores. Para ello se procedió a calcular el índice de polaridad de los sujetos adaptando la propuesta de Correa y Camacho (1993).

Teniendo en cuenta el índice de polaridad y el promedio de cada docente en cada uno de los factores se forman tres grupos de docentes: 25 (45,5\%) que asumen el primer factor, $17(30,9 \%)$ que asumen el primer y el tercer factor, y $13(23,6 \%)$ que asumen los tres factores identificados.

\subsection{INDICADORES SIGNIFICATIVOS}

A continuación se muestra el resumen de los indicadores significativos según los residuos tipificados corregidos (RTC) en cada factor (ver Tabla 2) que señalan si los factores están repartidos equilibradamente o no entre los valores que puede tener un ítem de acuerdo a lo establecido en Navas (2008).

Son especialmente interesantes los resultados obtenidos según la modalidad de la acción formativa: en el tercer factor es significativo el profesorado que imparte en modalidad Colectivos ( 6 docentes, $46,2 \%$ ) y también el que imparte en acciones formativas dirigidas a personas con discapacidad (4 docentes, 30,8\%). Estos últimos resultados muestran que los factores asumidos por el profesorado de la modalidad Formación e inserción son diferentes a los asumidos por el profesorado de la modalidad Colectivos. Igualmente, teniendo en cuenta que los Talleres FIL son la anterior denominación de la modalidad Colectivos, la afirmación anterior se refuerza puesto que también entre el profesorado que asume la orientación hacia lo social es significativo el que solo posee experiencia en Taller FIL (3 docentes, 23,1\%) y el que posee alguna experiencia en dichos talleres, independientemente de si ha tenido experiencia en otras modalidades de formación (10 docentes, 76,9\%).

Se ha realizado el análisis de conglomerados tanto bietápico como jerárquico, como técnica multivariante que agrupa casos (o sujetos) o variables, para conseguir la máxima homogeneidad en cada grupo/factor y la máxima diferencia entre los mismos, es decir, para ver cómo se agrupan los casos de la muestra. 
Tabla 2. Síntesis de las características significativas del profesorado de FPE en cada factor

\begin{tabular}{|c|c|c|c|c|}
\hline & Factor 1 & Factor 2 & Factor 3 & RTC \\
\hline Especialidad formativa: Actividades de venta & $4 / 16 \%$ & & & 2,3 \\
\hline Familia profesional: Comercio y Marketing & $4 / 16 \%$ & & & 2,3 \\
\hline Vinculación con la entidad en régimen de autónomos & $18 / 72 \%$ & & & 2,1 \\
\hline Entre 11 y 15 años de experiencia laboral & $10 / 43,5 \%$ & & & 2,8 \\
\hline $\begin{array}{l}\text { Experiencia en la impartición del módulo Igualdad } \\
\text { de género }\end{array}$ & $3 / 12 \%$ & & & 2 \\
\hline $\begin{array}{l}\text { Especialidad formativa: Montaje y mantenimiento de } \\
\text { instalaciones solares térmicas }\end{array}$ & & $5 / 29,4 \%$ & & 2,9 \\
\hline Familia profesional: Energía y Agua & & $5 / 29,4 \%$ & & 2,1 \\
\hline $\begin{array}{l}\text { Vinculación con la entidad con contrato de duración } \\
\text { definida }\end{array}$ & & $9 / 52,9 \%$ & & 1,9 \\
\hline Trabaja en otra entidad como docente & & $5 / 29,4 \%$ & & 2,1 \\
\hline Entre 26 y 30 años de experiencia laboral & & $2 / 11,8 \%$ & & 2,1 \\
\hline Menos de 5 años de experiencia docente & & $8 / 47,1 \%$ & & 2,2 \\
\hline $\begin{array}{l}\text { Consideran bastante importante el motivo de no } \\
\text { encontrar otro trabajo mejor }\end{array}$ & & $4 / 23,5 \%$ & & 1,9 \\
\hline Profesorado en la modalidad Colectivos & & & $6 / 46,2 \%$ & 2 \\
\hline Profesorado en la modalidad Colectivos: DIS & & & $4 / 30,8 \%$ & 1,9 \\
\hline Especialidad formativa: Pintor & & & $3 / 23,1 \%$ & 2 \\
\hline Entre 56 y 61 años de edad & & & $3 / 25 \%$ & 3,3 \\
\hline $\begin{array}{l}\text { No trabajan en otra entidad, ni como docente ni como } \\
\text { profesional }\end{array}$ & & & $11 / 84,6 \%$ & 1,9 \\
\hline $\begin{array}{l}\text { Experiencia solo en Taller de formación e inserción } \\
\text { laboral }\end{array}$ & & & $3 / 23,1 \%$ & 2,5 \\
\hline $\begin{array}{l}\text { Alguna experiencia en Taller de formación e inserción } \\
\text { laboral }\end{array}$ & & & $10 / 76,9 \%$ & 2,1 \\
\hline $\begin{array}{l}\text { Mucha importancia al motivo de no encontrar otro } \\
\text { trabajo mejor para seguir trabajando en la FPE }\end{array}$ & & & $5 / 41,7 \%$ & 4,3 \\
\hline Totales & $25 / 100 \%$ & $17 / 100 \%$ & $13 / 100 \%$ & \\
\hline
\end{tabular}




\subsection{INTERPRETACIÓN TEÓRICA}

Procedemos a continuación a interpretar los resultados y análisis estadísticos mediante el uso de la teoría. En este sentido, el análisis de contenido de los ítems que forman parte de cada factor nos recuerda a los enfoques de la enseñanza conceptualizados por Fenstermacher y Soltis (1998). El primer factor correspondería al enfoque terapeuta, el segundo al liberador y el tercero al ejecutivo.

A continuación se muestran las características de cada factor redactadas con términos autorreferenciales para, en posteriores investigaciones, facilitar la identificación o no con el mismo, es decir, para que el profesorado pueda señalar el grado de acuerdo que sostiene con los enunciados (su conocimiento atribucional):

- Características del profesorado que asume el primer factor (enfoque terapeuta): Creo que para que una acción formativa funcione de manera eficaz, hay que hacer una valoración adecuada de necesidades. Dichas necesidades, junto con los intereses del alumnado, los tengo en cuenta para definir los objetivos educativos. Creo que el alumnado aprende mejor ensayando y equivocándose, puesto que lo que el alumnado aprende experimentando, no lo olvida nunca. Durante mis clases, el alumnado está continuamente opinando y ocupado en algo y considero que la discusión en la clase es esencial para mantener una adecuada actividad de enseñanza. Creo que las relaciones en el aula deben ser plurales e iguales. En cuanto a la evaluación, suelo tener en cuenta si los trabajos elaborados por el alumnado van evolucionando a lo largo del curso, puesto que considero que lo fundamental es valorar no solamente el resultado, sino el conjunto de actividades realizadas por el alumnado.

- Características del profesorado que asume el segundo factor (enfoque liberador): En la acción formativa, el currículum responde y representa la ideología y cultura de la sociedad, puesto que el conocimiento que se imparte en la acción formativa implica nociones de poder y recursos económicos y de control social. Partiendo de que el fracaso escolar es producto más de las desigualdades sociales que de los métodos de enseñanza y de que no puede haber una auténtica igualdad de oportunidades mientras existan clases sociales, considero que la cultura que transmite la acción formativa aumenta las diferencias sociales. Durante las clases, la asamblea de alumnos/as y profesor/a es la que, realmente, regula la convivencia democrática. Por eso, previa discusión entre toda la clase, se seleccionan los textos y materiales para trabajar según los objetivos que hemos propuesto. Mi principal función como profesor/a es organizar la enseñanza para que el alumnado elabore su propio conocimiento.

- Características del profesorado que asume el tercer factor (enfoque ejecutivo): Considero que el conocimiento científico siempre es el más útil para enseñar. Mantengo las distancias con el alumnado para que me respete más y así tener menos problemas de disciplina. Considero que hay que forzar al alumnado para que aprenda puesto que, por sí mismo, no estudiaría. Así, durante las clases procuro que haya un cierto clima de competitividad entre el alumnado, porque mejora su motivación. Además, insisto en que me atienda en silencio y con interés puesto que considero que el alumnado disfruta más con una explicación mía que leyendo un libro o discutiendo en equipo. En cuanto a la evaluación, creo que es el único indicador fiable de la calidad de la enseñanza. 


\section{DISCUSIÓN DE RESULTADOS}

Se ha querido mostrar la realidad de los resultados obtenidos en los tratamientos estadísticos o procedimientos utilizados y el empeño por ser rigurosos en la investigación realizada, aunque los resultados estadísticos obtenidos no son los deseados ni los esperados en una investigación. Otras investigaciones sobre teorías implícitas, según la información que consta en los informes a los que se ha accedido, recurren a los análisis de polaridad (Martel, 2009) junto al análisis de tipicidad de los ítems (Jiménez, 2002, 2005, 2009; Jiménez \& Cabrera, 1999; Jiménez \& Correa, 2002, 2003; Makuc, 2011) sin mencionar la fiabilidad de la escala de teorías implícitas. Tan solo Serrano (2010), Gómez y Guerra (2012) y Martínez et al. (2014) señalan la fiabilidad del instrumento.

Ante los resultados presentados, podemos decir que las factorizaciones realizadas abocan hacia una estructura factorial de tres componentes que posee resultados estadísticos aceptables y con sentido teórico para el contexto de la FPE. En primer lugar, poseen una fiabilidad moderada aceptable y sí hay sujetos que aparecen polarizados, es decir, el resultado estadístico es aceptable. Y en segundo lugar, el análisis de polaridad junto con el análisis de contenido de los factores permite identificar lo que se ha denominado orientaciones de la enseñanza, puesto que las teorías implícitas orientan las acciones de las personas (Makuc, 2011, 2015). Dichas orientaciones, además de poseer mejores resultados estadísticos, también poseen sentido teórico para la muestra: orientación hacia el participante, orientación hacia la docencia y orientación hacia lo social.

El grupo de docentes que asume el primer factor sería un ejemplo de la orientación centrada en el participante cuyo centro es el propio alumnado. El grupo de docentes que asume tanto el primer factor como el tercero, sería un ejemplo de la orientación centrada en la docencia, puesto que sigue centrado en el participante, pero introduce la labor del propio docente como clave en el proceso de enseñanza-aprendizaje. El tercer grupo de docentes que asume las características de los tres factores, es decir, contempla tanto al participante como al profesorado añadiendo la clave de lo social, asume la llamada orientación hacia lo social. Son tres maneras de entender la enseñanza que amplían la visión que sobre ella puede tenerse y que reflejan la orientación hacia un aspecto del proceso de enseñanzaaprendizaje coherente con el contexto de la FPE. Pero no se pueden considerar teorías (el carácter explícito o implícito se deberá confirmar con otra metodología de investigación).

De manera exploratoria, se pueden representar las orientaciones de la enseñanza del profesorado de FPE como se muestra en la Figura 1: 
Figura 1. Las orientaciones de la enseñanza en la FPE: relación entre objetivos y elemento central

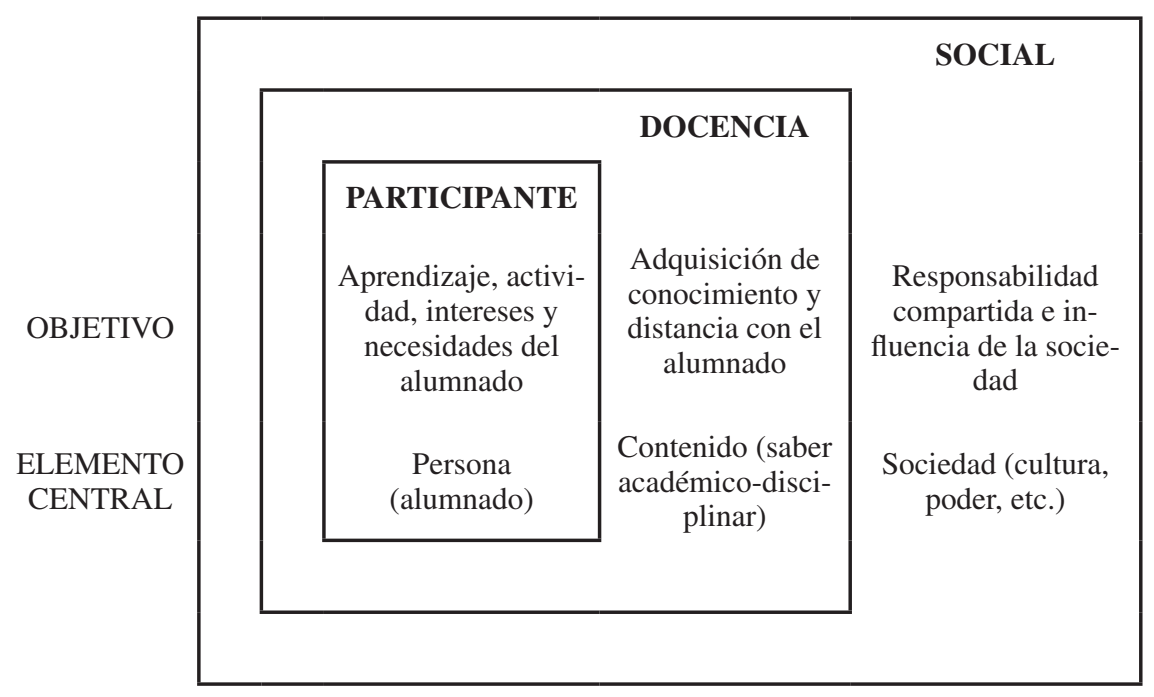

Fuente: Ros-Garrido (2014, p. 517)

Esta clasificación tiene sentido por dos motivos. En primer lugar, el resultado concuerda en cierta manera con resultados obtenidos en la clasificación de las teorías implícitas identificadas por Marrero (1988a) en los que el 85,45\% del profesorado asumía la teoría expresiva, puesto que 5 de los 9 ítems que forman parte del primer factor pertenecen a la dicha teoría. En segundo lugar, en otras investigaciones realizadas en otros contextos (alumnado de pedagogía o magisterio, profesorado de educación infantil, primaria y superior) la teoría expresiva también es la más asumida por el profesorado participante (Alarcón \& Reyno, 2009; Ferrández et al., 2000; Jiménez, 2002; Jiménez \& Correa, 2002; Martínez, 2009; Zapata, 2012). Así, las teorías implícitas sustentan la visión que los formadores tienen sobre sus estudiantes (Gimeno, 2010b). Aplicando al contexto de la FPE las palabras de Gimeno (2010a) para apreciar lo que se consigue desde una teoría del currículum, el centro de gravedad de atención debe desplazarse de la enseñanza al aprendizaje, de los que enseñan a quienes aprenden, de lo que se pretende lograr a lo que se logra en realidad, de las intenciones declaradas a los hechos logrados. Por lo tanto, a la luz de los resultados, para el profesorado de FPE el alumnado es el centro de gravedad.

En el Cuadro 1 se refleja la coherencia entre las orientaciones de la enseñanza y los factores identificados en la investigación, así como la relación con los enfoques de la enseñanza conceptualizados por Fenstermacher y Soltis (1998), las teorías implícitas y las teorías de la enseñanza identificadas por Marrero (1988a) y las racionalidades de la práctica pedagógica según Carr y Kemmis (1988) y Carr (1990). Las teorías implícitas y las teorías de la enseñanza acompañadas con un "+" refieren las que más destacan dentro de la orientación de la enseñanza, es decir, hay más ítems que originalmente pertenecían a dicha teoría implícita o teoría de enseñanza. 
Estudios Pedagógicos XLIII, Nº 1: 269-288, 2017 TEORÍAS IMPLÍCITAS DEL PROFESORADO DE FORMACIÓN PROFESIONAL PARA EL EMPLEO

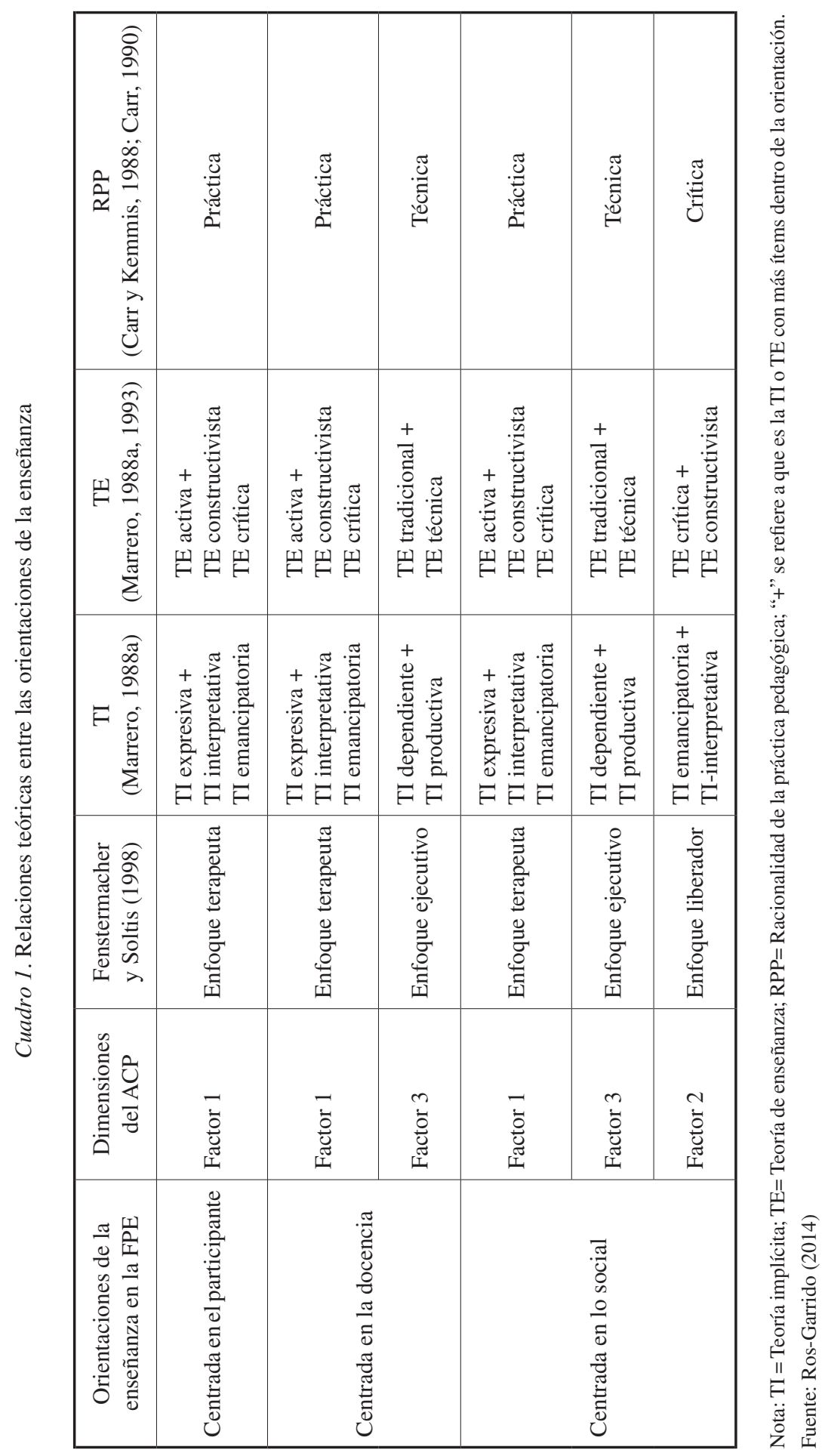


Para finalizar este apartado, hacemos referencia a los indicadores que han formado parte de la investigación. Sobre los indicadores que no han obtenido resultados significativos según los RTC en las orientaciones de la enseñanza, en principio, no sería necesario seguir investigando (sexo, titulación o especialidad académica y nivel de estudios del profesorado, tiempo que lleva trabajando en la entidad, motivo por el que cree que le eligió la entidad, sector profesional y nivel de cualificación al que pertenece la acción formativa que imparte el profesorado). En especial sobre el tipo de estudios porque en otra investigación (Serrano, 2010), aunque realizada en el contexto universitario, tampoco se ha verificado que esta variable incida en las creencias de dicho profesorado.

Sobre los indicadores que sí han permitido diferenciar entre la asunción de una u otra orientación se debería seguir profundizando (la edad del profesorado, si trabaja en otra entidad, los años y el ámbito de experiencia docente, los años de experiencia laboral del profesorado, la experiencia en la impartición de ciertos módulos, los módulos impartidos durante la acción formativa, la importancia de la formación complementaria, la consideración de los saberes y desempeños y el tiempo dedicado y la importancia de las tareas realizadas durante la acción formativa, la modalidad y el colectivo al que va dirigida la acción formativa, la especialidad formativa y familia profesional de la acción formativa que imparte, los objetivos que se alcanzan en la acción formativa, la importancia de la experiencia que debe poseer el profesorado, los documentos que orientan y guían tanto la práctica formativa como evaluadora). Estos últimos indicadores se deberían tener en cuenta en posteriores investigaciones puesto que son significativos entre el profesorado que asume una determinada orientación de la enseñanza. Sin embargo, por ejemplo recurriendo de nuevo a la investigación de Serrano (2010), la antigüedad en la docencia, en este caso universitaria, no incide en las creencias de dicho profesorado, aunque Gómez y Guerra (2012) sí encuentran resultados interesantes en los profesores con menos experiencia docente (hasta 6 años), donde predomina un enfoque constructivista.

Por lo tanto, es necesario seguir profundizando qué indicadores o variables influyen o no en las orientaciones de la enseñanza (o teorías implícitas) asumidas por el profesorado.

\section{CONCLUSIONES}

Las investigaciones realizadas por Marrero (1988a, 1993) y los enfoques de la enseñanza conceptualizados por Fenstermacher y Soltis (1998) han proporcionado elementos clave a la investigación. El primero, una conceptualización de las concepciones sobre la enseñanza (teorías de enseñanza) del profesorado y un instrumento que permite aproximarse a su asunción (teorías implícitas). El segundo, un marco de referencia amplio (perspectivas curriculares) que permite aproximarse a la enseñanza más allá de lo escolar y que es válido para la FPE, que contempla los elementos que están presentes en cualquier proceso de enseñanza y aprendizaje, en el que la finalidad del proceso define cada uno de los enfoques de la enseñanza (Fenstermacher \& Soltis, 1998).

Por una parte, Martel (2009) sostiene que los supuestos en los que se basan las teorías de la enseñanza propuestas para el estudio de las teorías implícitas del profesorado corresponden a una concepción masculina con las que las mujeres no se identifican. Nuestra suposición es que, del mismo modo, dichos supuestos corresponden a la escolaridad obligatoria, por lo que tal vez no sean válidos para una formación orientada al empleo. 
Por otra parte, se confirma que el profesorado de FPE no tiene una única teoría implícita dominante, sino que son multivariadas y no atienden a un único paradigma ideológico (Sosa \& Tejada, 1996), es decir, el profesorado asume elementos de varias teorías implícitas. Aunque es posible encontrar un sustrato común entre la constelación de concepciones y creencias: el profesorado de FPE asume al participante como centro de la enseñanza, es decir, asume en mayor medida la teoría expresiva o, utilizando los enfoques de Fenstermacher y Soltis (1998), el enfoque terapeuta.

En la Figura 2 se ha representado cómo sería la relación entre las tres orientaciones de la enseñanza en la práctica. El centro es el mismo, el participante. Los márgenes no son impermeables sino que, dependiendo de las demandas del contexto y de las exigencias de las tareas, el profesorado podría ir modificando sus prácticas llegando a desarrollar aquellas que se considerarían propias de otra orientación de la enseñanza. De ahí que los márgenes no sean líneas continuas y que la distancia entre los márgenes de las orientaciones pueda llegar a ser muy estrecha, lo que en la práctica se traduciría en realizar prácticas diferentes a la orientación asumida. Las formas irregulares de las líneas también representan que la orientación asumida por el profesorado no es un marco rígido y uniforme. En cualquier caso, esta representación de las orientaciones no es concluyente, sino que obedece a los resultados de la investigación aquí presentada; es conveniente seguir investigando para confirmarlos y ver si el profesorado modifica las prácticas que desarrolla dependiendo de las demandas de la tarea y del contexto.

Figura 2. Representación de las orientaciones de la enseñanza en la FPE

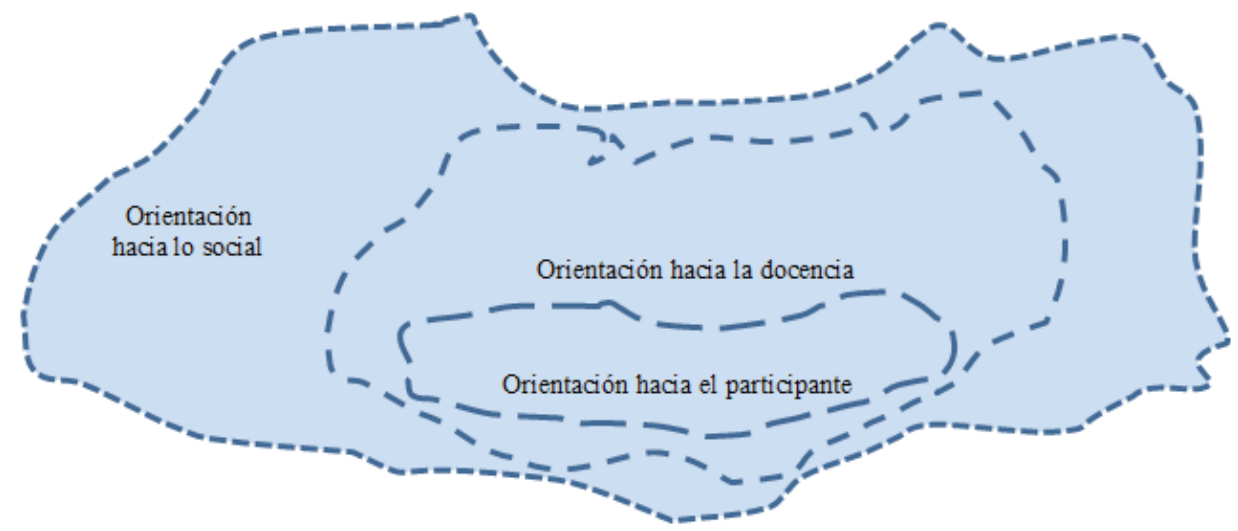

Fuente: Ros-Garrido (2014)

Así pues, la investigación realizada con el establecimiento de las orientaciones de la enseñanza identificadas en el profesorado de FPE permite concluir que las teorías implícitas $\mathrm{u}$ orientaciones de la enseñanza de dicho profesorado son diferentes dependiendo del colectivo al que van dirigidas las acciones formativas, puesto que el profesorado de la modalidad Colectivos asume en mayor medida la orientación hacia lo social (enfoque liberador, racionalidad crítica o teoría emancipatoria). 
La significatividad obtenida en el profesorado de la modalidad Colectivos que asume la orientación hacia lo social se confirma con los resultados del ámbito de la experiencia docente: la experiencia en los Talleres FIL (anterior denominación de la modalidad Colectivos), tanto en exclusiva como también en otro tipo de acciones formativas, destaca en dicha orientación. El resultado está relacionado con la formación pedagógica como requisito establecido en la norma que regula estas acciones formativas. Allí se estipula que deben desarrollarse tanto por una persona experta en los módulos de FPE que acredite conocimientos teórico-prácticos como por una persona que coordine y posea la formación de Licenciatura/Grado en Psicología, Pedagogía o Psicopedagogía, Ciencias de la Educación, Doctor en Educación Social, Trabajo Social o Magisterio, o Técnico Superior en Integración Social. Asimismo, en función de las características de los participantes, pueden intervenir otros profesionales. Esta conclusión es coherente con la obtenida por Barrios (2015), puesto que el programa de formación de dichos docentes (estrategias y contenidos de formación) influye en sus creencias.

Por lo tanto, las características de los participantes como personas en situación o riesgo de exclusión social y como personas con alguna discapacidad (y no solo como personas desempleadas que pertenecen a colectivos con especiales dificultades de inserción en el mercado de trabajo) están relacionadas con que el profesorado tenga una mayor consideración de los aspectos sociales que influyen en sus procesos de enseñanzaaprendizaje con estos colectivos. Falta averiguar si la orientación se asume previamente o si se asume como consecuencia de las experiencias en dicha modalidad de formación, aunque de acuerdo a los resultados obtenidos por Baena (2009) y por Castro (2009) las teorías implícitas más progresistas (interpretativa y emancipatoria) son independientes del contexto.

En síntesis, las aportaciones teóricas de los enfoques de la enseñanza llevan a concluir que "lo que hacemos como docentes es el resultado de la influencia combinada de 4 factores: nosotros mismos, los estudiantes, el contenido y el contexto" (Fenstermacher \& Soltis, 1998, p. 153). Sin embargo, para el profesorado de la FPE parece que lo más importante es el alumnado o participante, seguido del propio profesorado y del contexto o sociedad. Así pues, el contenido no parece relevante; lo que confirma que el currículum en la FPE es cerrado (Ros-Garrido, Marhuenda-Fluixá \& Navas-Saurin, 2016).

\section{PROSPECTIVA}

Los resultados obtenidos deben considerarse preliminares y la identificación de las tres orientaciones de la enseñanza en la FPE constituye un marco de referencia en investigaciones futuras y deben incluirse en los procesos de formación del profesorado para que este pueda explicitar, analizar y profundizar en las teorías implícitas que orientan sus decisiones y acciones.

Las investigaciones futuras necesariamente deben incluir algún instrumento de carácter cualitativo que permita complementar y corroborar los resultados obtenidos cuantitativamente (entrevistas semi-estructuradas al profesorado, alguna entrevista en profundidad o historia de vida de algún profesor o profesora en concreto o el estudio de caso de alguna entidad o, al menos, introducir alguna pregunta abierta en los cuestionarios para obtener información de carácter cualitativo). 
Puesto que "la manera de entender la docencia tendrá un gran efecto en lo que haga el docente" (Fenstermacher \& Soltis, 1998, p. 15) es necesario seguir avanzando en la investigación sobre la FPE, tanto sobre el conocimiento docente como desde el conocimiento docente, y teniendo en cuenta a los agentes implicados en la misma. Es decir, queda pendiente profundizar en las orientaciones de la enseñanza identificadas (teorías implícitas o nivel atribucional) y su correspondencia con la práctica docente en un aula de FPE así como tener en cuenta otras variables, como la cultura del centro o entidades que desarrollan FPE, para seguir generando resultados académicos que puedan contribuir a la comprensión de la praxis del profesorado de FPE.

\section{REFERENCIAS BIBLIOGRÁFICAS}

Alarcón, T. I., \& Reyno, A. (2009). Teorías implícitas de la Enseñanza en la Educación Física: estudio longitudinal, 2005-2008. Revista des-encuentros, 8(1), 37-47.

Álvarez, V., García-Gómez, M. S., Gil-Flores, J., \& Romero, S. (2015). Necesidades de información y orientación del alumnado de formación profesional en la comunidad autónoma de Andalucía. Bordón. Revista de Pedagogía, 67(3), 15-34.

Angulo, F. (1999). De la investigación sobre la enseñanza al conocimiento docente. En A. Pérez, J. Barquín \& J. F. Angulo (Eds.), Desarrollo profesional del docente: política, investigación y práctica (pp. 261-319). Madrid: Akal.

Azofra, M. J. (1999). Cuestionarios. Madrid: Centro de Investigaciones Sociológicas.

Baena, M. D. (2009). La reconstrucción de la práctica desde las tareas académicas y las teorías implícitas del profesorado. En J. Marrero (Ed.), El pensamiento reencontrado (pp. 159-185). Barcelona: Octaedro.

Barrientos, C., \& Navío, A. (2015). Formador de la educación superior técnico profesional: Percepciones sobre su trabajo docente. Estudios pedagógicos, 41(1), 45-61.

Barrios, E. (2015). Evolución de las creencias del profesorado en formación sobre la enseñanza y el aprendizaje del inglés. Bordón. Revista de Pedagogía, 67(2), 45-62.

Buendía, L., \& Berrocal, E. (2008). Evaluación de un programa de Formación Ocupacional para la inserción laboral. Revista de Investigación Educativa, 26(1), 157-190.

Buendía, L., Expósito, J., \& Sánchez, M. (2012). Investigación evaluativa de formación profesional para el empleo en el ámbito local. Revista de Investigación Educativa, 30(1), 161-179. doi:10.6018/rie.30.1.115971

Campo-Arias, A., \& Oviedo, H. C. (2008). Propiedades psicométricas de una escala: la consistencia interna. Revista Salud Pública, 10(5), 831-839.

Carr, W. (1990). Cambio educativo y desarrollo profesional. Investigación en la escuela, 11, 3-12.

Carr, W., \& Kemmis, S. (1988). Teoría crítica de la enseñanza. La investigación-acción en la formación del profesorado. Barcelona: Martínez Roca.

Castro, F. (2009). Las teorías implícitas del profesorado de secundaria. Un análisis de la relación entre culturas de centro y teorías implícitas en enseñanza secundaria. En J. Marrero (Ed.), El pensamiento reencontrado (pp. 116-135). Barcelona: Octaedro.

Cepeda, O. (2009). La «reflexión» y las teorías implícitas del profesorado: un análisis de las concepciones del profesorado de formación inicial. En J. Marrero (Ed.), El pensamiento reencontrado (pp. 218-245). Barcelona: Octaedro.

Correa, A. D., \& Camacho, J. (1993). Diseño de una metodología para el estudio de las teorías implícitas. En M. J. Rodrigo, A. Rodríguez \& J. Marrero, Las teorías implícitas. Una aproximación al conocimiento cotidiano (pp. 123-165). Madrid: Visor.

Correa, A. D., \& Marrero, J. (1992). Las teorías implícitas como marco de estudio del pensamiento 
del profesor: Descripción de una metodología de investigación. En A. Estebaranz \& V. Sánchez (Eds.), Pensamiento de profesores y desarrollo profesional (I) Conocimiento y teorías implícitas (pp. 57-69). Sevilla: Universidad de Sevilla.

Fenstermacher, G. D., \& Soltis, J. F. (1998). Enfoques de la enseñanza. Buenos Aires: Amorrortu.

Ferrández, A., Tejada, J., Jurado, P., Navío, A., \& Ruiz, C. (2000). El formador de formación profesional y ocupacional. Barcelona: Octaedro.

Gimeno, J. (2010a). ¿Qué significa el curriculum? En J. Gimeno (Comp.), Saberes e incertidumbres sobre el curriculum (pp. 21-43). Madrid: Morata.

Gimeno, J. (2010b). La carrera profesional para el profesorado. Revista Interuniversitaria de Formación de Profesorado, 24(2), 243-260.

Gómez, V., \& Guerra, P. (2012). Teorías implícitas respecto a la enseñanza y el aprendizaje: ¿Existen diferencias entre profesores en ejercicio y estudiantes de pedagogía? Estudios pedagógicos, $38(1), 25-43$.

Jiménez, A. B. (2002). Nivel educativo y teorías implícitas sobre la enseñanza: estructura de creencias en los docentes de educación infantil y primaria, secundaria y superior (Tesis doctoral). Universidad de La Laguna. Recuperado de http://hdl.handle.net/11162/27990

Jiménez, A. B. (2005). Las teorías implícitas del profesorado sobre los distintos ámbitos del proceso enseñanza-aprendizaje: la interacción profesor-alumno en los tres niveles educativos. Qurriculum: Revista de teoría, investigación y práctica educativa, 18, 211-236.

Jiménez, A. B. (2009). Un contraste de ideas entre niveles educativos. Las teorías implícitas de los profesores de educación infantil y primaria, secundaria y superior. En J. Marrero (Ed.), El pensamiento reencontrado (pp. 46-93). Barcelona: Octaedro.

Jiménez, A. B., \& Cabrera, L. (1999). Aproximación a las teorías implícitas del profesorado de Educación Infantil y Primaria, Secundaria y Superior sobre los medios de enseñanza. Revista Píxel-Bit. Revista de medios y educación, 13, 47-58.

Jiménez, A. B., \& Correa, A. D. (2002). El modelo de teorías implícitas en el análisis de la estructura de creencias del profesorado universitario sobre la enseñanza. Revista de Investigación Educativa, 20(2), 525-548.

Jiménez, A. B., \& Correa, A. D. (2003). Concepciones del profesorado de educación infantil y primaria, secundaria y superior sobre la disciplina y gestión del aula. Qurriculum: Revista de teoría, investigación y práctica educativa, 16, 87-104.

Kagan, D. (1992). Professional Growth Among Preservice and Beginning Teachers. Review of Educational Research, 62(2), 129-170.

Makuc, M. (2008). Teorías implícitas de los profesores acerca de la comprensión de textos. Revista signos, 41(68), 403-422.

Makuc, M. (2011). Teorías implícitas sobre comprensión textual y la competencia lectora de estudiantes de primer año de la Universidad de Magallanes. Estudios pedagógicos, 37(1), 237-254.

Makuc, M. (2015). Las teorías implícitas sobre la comprensión textual y las estrategias metacognitivas de estudiantes universitarios de primer año. Estudios pedagógicos, 41(1), 143-166.

Marhuenda, F. (2012). La Formación Profesional. Madrid: Síntesis.

Marrero, J. (1988a). Teorías implícitas y planificación del profesor (Tesis Doctoral). Universidad de La Laguna, Tenerife.

Marrero, J. (1988b). Las teorías implícitas y la planificación de la enseñanza. En C. Marcelo (Ed.), Avances en el estudio del pensamiento de los profesores (pp. 135-144). Sevilla: Servicio de Publicaciones de la Universidad de Sevilla.

Marrero, J. (1991). Teorías implícitas del profesorado y currículum. Cuadernos de pedagogía, 197, 66-69.

Marrero, J. (1992). Teorías implícitas del profesorado: un puente entre la cultura y la práctica de la enseñanza. En A. Estebaranz \& V. Sánchez (Eds.), Pensamiento del profesor y desarrollo profesional (I). Conocimiento y Teorías Implícitas (pp. 9-21). Sevilla: Universidad de Sevilla. 
Marrero, J. (1993). Las teorías implícitas del profesorado: Vínculo entre la cultura y la práctica de la enseñanza. En M. J. Rodrigo, A. Rodríguez \& J. Marrero (Eds.), Las teorías implícitas. Una aproximación al conocimiento cotidiano (pp. 243-276) Madrid: Visor.

Marrero, J. (2009). Escenarios, saberes y teorías implícitas del profesorado. En J. Marrero (Ed.), El pensamiento reencontrado (pp. 8-44). Barcelona: Octaedro.

Marrero, J. (2010). El currículum que es interpretado. ¿Qué enseñan los centros y los profesores y profesoras? En J. Gimeno (Coord.), Saberes e incertidumbres sobre el currículum (pp. 221-245). Madrid: Morata.

Martel, M. C. (2009). ¿Hombres críticos y mujeres dependientes? Una aproximación al pensamiento del profesorado desde una perspectiva de género. En J. Marrero (Ed.), El pensamiento reencontrado (pp. 94-115). Barcelona: Octaedro.

Martínez, J. F. (2009). Las teorías implícitas como conocimiento práctico del profesorado en formación inicial. En J. Marrero (Ed.), El pensamiento reencontrado (pp. 204-217). Barcelona: Octaedro.

Martínez, J. F., Zarate, A., Salazar, B. P., \& Palacios, A. (2014). Concepciones en torno de la enseñanza docente. El caso de la Universidad Autónoma de San Luis Potosí. Revista Intercontinental de Psicología y Educación, 16(1), 153-173.

Merino, R. (2012). Editorial. Revista de la Asociación de Sociología de la Educación, 5(1), 3-4.

Molpeceres, M.A., Chulvi, B., \& Bernad, J.C. (2004). Concepciones sobre la enseñanza y prácticas docentes en un sistema educativo en transformación: un análisis en los PGS. En M. A. Molpeceres (Coord.), Identidades y formación para el trabajo (pp. 141-196). Montevideo: Cinterfor.

Navas, A. A. (2008). Estudio de la práctica pedagógica en garantía social (Tesis de doctorado, Universitat de València). Recuperada de http://roderic.uv.es/handle/10550/15721.

OCDE. (2011). Preparándose para trabajar. Barcelona: Fundación Barcelona FP. Recuperado de http://www.redetis.iipe.unesco.org/publicaciones/ocdept.pdf

Olmos, P. (2014). Competencias básicas y procesos perceptivos: factores claves en la formación y orientación de los jóvenes en riesgo de exclusión educativa y sociolaboral. Revista de Investigación Educativa, 32(2), 531-546.

Oramas, A. (2013). Creencias sobre la enseñanza que poseen docentes en la modalidad a distancia/ Teachers' beliefs on distance education. Una Investig@ ción, 5(9), 38-58.

Orden 29/2012, de 22 de junio, de la Conselleria de Educación Formación y Empleo, por la que se determina el Programa de Formación Profesional para el Empleo y se regulan y convocan subvenciones para la realización de acciones formativas dirigidas prioritariamente a los trabajadores desempleados durante el ejercicio de 2012. (DOGV 27 de junio de 2012).

Pérez, C. (2005). Técnicas Estadísticas con SPSS 12. Aplicaciones al análisis de datos. Madrid: Editorial Pearson Prentice Hall.

Pozo, J. I., Scheuer, N., Mateos, M., \& Pérez, M. P. (2006). Las teorías implícitas sobre el aprendizaje y la enseñanza. En J. I. Pozo, N. Scheuer, M. P. Pérez, M. Mateos, E. Martín \& M. Cruz, Nuevas formas de pensar la enseñanza y el aprendizaje: las concepciones de profesores y alumnos (pp. 95-132). Barcelona: Grao.

Real Decreto 395/2007, de 23 de marzo, por el que se regula el subsistema de formación profesional para el empleo (BOE de 11 de abril de 2007).

Rodrigo, M. J., Rodríguez, A., \& Marrero, J. (1993). Las teorías implícitas. Una aproximación al conocimiento cotidiano. Madrid: Visor.

Rodríguez, E. (2003). Teorías implícitas del Profesorado y Modelos de Formación Docente. Contexto educativo: revista digital de investigación y nuevas tecnologías. 28, 1-8.

Ros-Garrido, A. (2014). Teorías implícitas y concepciones de planificación en la formación profesional para el empleo. Análisis en la provincia de Valencia (Tesis doctoral). Universidad de Valencia, Valencia. Recuperado de http://roderic.uv.es/handle/10550/41186

Ros-Garrido, A., Marhuenda-Fluixá, F., \& Navas-Saurin, A. A. (2016). La naturaleza del curriculum 
en la formación profesional para el empleo y los documentos que orientan su práctica. Libro de actas digital CIMIE-2015. Recuperado de http://amieedu.org/actascimie15/wp-content/ uploads/2016/06/Contribution352.pdf

Ruiz, C. (2001). La evaluación de programas de formación de formadores en el contexto de la formación en y para la empresa (Tesis Doctoral). Universidad Autónoma de Barcelona, Barcelona. Recuperado de http://hdl.handle.net/10803/5003

Serrano, R. C. (2010). Pensamientos del profesor: un acercamiento a las creencias y concepciones sobre el proceso de enseñanza-aprendizaje en la Educación Superior. Revista de Educación, 352, 267-287.

Sosa, F., \& Tejada, J. (1996). Las teorías implícitas en la formación ocupacional y profesional. Educar, 20, 105-116.

Zapata, M. (2012). Teorías implícitas de los docentes sobre enseñanza y su producción pedagógica (Tesis doctoral). Universidad Central de Venezuela, Venezuela. Recuperado de http://saber.ucv. ve/jspui/handle/123456789/2115 